\title{
The Correlation Between Stroke and Coronavirus Disease (COVID-19): Where is the Evidence?
}

The Editor's Pick for this issue of EMJ is the fantastic investigative review by Pittams et al., which aims to understand the role that the coronavirus disease (COVID-19) plays in stroke aetiology. As a multifactorial disease, many of the modifiable and genetic risk factors of stroke have been well researched, yet still undetermined is the role played by infectious diseases in stroke pathophysiology. Further insight into the interplay between these two disease entities is vital both for understanding stroke aetiology and advancing the therapies needed to fight pathogen-induced stroke.
Authors:
Ashleigh Pittams, ${ }^{1,2}$ Ariana Axiaq, ${ }^{3}$ Amna Qamar, ${ }^{4}$ Bianca Botezatu, ${ }^{3}$
*Amer Harky ${ }^{5-7}$
1. Royal Sussex County Hospital, Brighton, UK
2. Sussex University Hospitals NHS Trust, Brighton, UK
3. School of Medicine, Dentistry and Biomedical Sciences, Queen's University Belfast, Belfast, Northern Ireland
4. School of Medicine, University of Liverpool, Liverpool, UK
5. Department of Cardiothoracic Surgery, Liverpool Heart and Chest Hospital, Liverpool, UK
6. Department of Integrative Biology, Faculty of Life Sciences, University of Liverpool, Liverpool, UK
7. Liverpool Centre for Cardiovascular Science, University of Liverpool and Liverpool Heart and Chest Hospital, Liverpool, UK
*Correspondence to aaharky@gmail.com

Disclosure:

The authors have declared no conflicts of interest.

Received:

Accepted:

Keywords:

Citation:
25.07.20

21.10 .20

Brain, cerebrovascular accident, infection, nervous system.

EMJ. 2021; DOI/10.33590/emj/20-00184

\begin{abstract}
Stroke is the second leading cause of death globally. Despite the decreasing trend in stroke mortality, its incidence and prevalence follow an upwards trajectory that is envisaged to continue for years to come. Previous literature has suggested a role for infectious disease in stroke aetiology; however, the pathophysiological basis for this has never fully been understood. Emerging infections, such as coronavirus disease (COVID-19), present new challenges that must be addressed, to prevent them from contributing to the predicted rise in stroke incidence. Almost one in 20 patients diagnosed with COVID-19 experience a stroke thereafter, hence achieving better understanding of the interactions between these disease entities is of major clinical significance.
\end{abstract}




\section{INTRODUCTION}

Stroke is a cerebrovascular disease, whereby blockage or rupture of a cerebral artery results in cerebral infarction or haemorrhage, respectively. It is the second most common cause of death globally, accounting for 80.5 deaths per 100,000 population. ${ }^{2}$ While the mortality rate of stroke appears to be decreasing, ${ }^{2}$ its prevalence is envisaged to rise over coming years, with a predicted increase in both stroke incidence and survival contributing to this. ${ }^{3}$ Its multifactorial nature means that many of the modifiable and genetic risk factors implicated in stroke aetiology have been well researched. However, the role of infectious agents in provoking cerebrovascular events has often been undermined. ${ }^{4,5}$

The relationship between stroke and infectious disease is complex. The neurological sequelae of stroke and infectious diseases may overlap, creating diagnostic uncertainty, ${ }^{6}$ and prior stroke is a poor prognosticator in coronavirus disease (COVID-19) infection.7 Additionally, several infectious agents have been directly implicated in the development of stroke. ${ }^{8-10}$ Some of these remain poorly investigated. This is particularly true for emerging infections, such as COVID-19, which pose a new opportunity to understand stroke pathophysiology.

Several mechanisms have been implicated in stroke secondary to infection.11-14 However, stroke in patients with COVID-19 is likely the result of several processes acting synergistically, rather than a single mechanism in isolation. Treatments for pathogen-induced stroke should be centred around counteracting these pathological pathways. However, without adequate understanding of the pathophysiology of stroke in infectious disease, it is difficult to optimise a management plan for when the two occur simultaneously. In this review, the authors aim to better understand the pathophysiology of stroke in infectious disease, and in particular COVID-19 infection. The authors hope that this will enable management and treatment strategies to become nuanced, optimising the care of patients experiencing a stroke following COVID-19 infection.

\section{METHODS}

The databases searched included MEDLINE, SCOPUS, Embase, Cochrane Library, and Google Scholar. The review question had two main parts: 1) pathophysiological mechanisms of stroke in infection; and 2) management strategies for patients with concurrent infection and stroke. A separate search was conducted for each of these. If the search retrieved papers applicable to the entire article, the results were shared between different parts of this review. Keywords included "stroke", "cerebrovascular accident", "brain", "nervous system", "infection”, "COVID-19", "coronavirus", "SARS-CoV-2", and "infectious disease". Part 1 also included "pathophysiology", "cause", and "trigger", while Part 2 included "treatment", "management", and "strategies". After reviewing the search results, some search terms were restricted to title and abstract fields. Each of the search terms were imputed as keywords and then combined as MeSH terms to ensure a comprehensive search. The last literature search was on $23^{\text {rd }}$ July 2020.

All articles retrieved were independently screened by two authors, and consensus was reached by consulting the senior author if there were any discrepancies. Studies met the inclusion criteria if they discussed stroke and infection with respect to pathophysiology and treatment. The main exclusion criteria were non-English language studies, editorials, commentaries, and studies for which the full text was unavailable. No restriction was placed on publication year to ensure all relevant studies were captured. The search results are presented in a narrative manner throughout this article and a summary table is displayed in the pathophysiology section of the results.

\section{PATHOPHYSIOLOGY OF STROKE IN INFECTIOUS DISEASE}

In recent years, some great insight into the association between infection and stroke has been achieved. However, the true extent of this has proved difficult to determine because of the differences in definitions and criteria for stroke and infection between studies. Various mechanisms have been proposed with two main themes, as outlined in Table 1.15-23 
Table 1: Brief overview of the proposed pathophysiological mechanisms of stroke in viral infections, including coronavirus disease (COVID-19).

\begin{tabular}{|c|c|c|}
\hline \multicolumn{2}{|l|}{ Proposed mechanisms } & \multirow{2}{*}{$\begin{array}{l}\text { Supporting evidence } \\
\text { Van der Poll et al.,15 } 1990 \\
\text { Manousakis et al.,16 } 2009\end{array}$} \\
\hline Inflammation & Increased levels of TNFa & \\
\hline & Increased levels of IL-6 & $\begin{array}{l}\text { Huber et al.,, } 1999 \\
\text { Amlie-Lefond et al., }{ }^{18} 2016\end{array}$ \\
\hline & Increased circulating leukocytes & Rivers et al.,.9 1975 \\
\hline & $\begin{array}{l}\text { Inhibition of anticoagulant factors, e.g., } \\
\text { proteins C and S }\end{array}$ & $\begin{array}{l}\text { Esmon et al., }{ }^{20} 1991 \\
\text { Hesselvik JF et al.,21 } 1991\end{array}$ \\
\hline \multirow[t]{3}{*}{ Direct causation (selected examples) } & HIV & $\begin{array}{l}\text { Ortiz G et al..,22 } 2007 \\
\text { Miller EC et al., }{ }^{23} 2016\end{array}$ \\
\hline & Herpes-zoster virus & Miller EC et al., ${ }^{23} 2016$ \\
\hline & Varicella-zoster virus & Miller EC et al., ${ }^{23} 2016$ \\
\hline
\end{tabular}

First, inflammation may increase stroke tendency. Increased levels of inflammatory mediators have been detected in stroke patients following infection, compared with those without; ${ }^{24}$ suggesting that activation of inflammatory pathways may in turn contribute towards a procoagulant state. It is thought that inflammation can give rise to increased atherosclerosis, plaque rupture, and thrombosis, eventually leading to ischaemic stroke. ${ }^{9}$ Infarction may also be a result of cerebral vasculitis; this has previously been reported in patients with tuberculosis, meningitis, and syphilis. ${ }^{9,24}$

There are several pathways that have been suggested to increase coagulation when stimulated by inflammation. Rivers et al. ${ }^{19}$ reported that endotoxin-induced monocytes promote coagulation through their expression of thromboplastin. Several cytokines, such as TNFa, have also been implicated in activating the common pathway of coagulation in severe infection..$^{15}$ TNF $\propto$ may also be procoagulant due to its inhibition of the fibrinolytic system. ${ }^{16}$

Similarly, one of the main cytokines that has been highlighted as a key player in the disease progression, from infection to stroke, is IL-6. In murine models, the presence of IL-6 increased the progression of fatty atheromas found in atherosclerosis, a major risk factor for stroke. ${ }^{17}$
Alongside promotion of procoagulant factors, there may be concurrent inhibition of the anticoagulant modulators proteins $C$ and $S .{ }^{20,21}$ Ortiz et al. ${ }^{22}$ agreed that protein $\mathrm{S}$ deficiency may be an additive mechanism for initiating infarction. There is accumulating evidence that infection-induced inflammation and subsequent procoagulant state is one of the main mechanisms behind stroke postinfection. However, the specific pathways involved in this process are yet to be isolated and so further investigation is required.

Haemorrhagic strokes postinfection are investigated less frequently. One case-crossover study reported that haemorrhagic strokes were primarily associated with preceding urinary tract infection, respiratory infection, and sepsis. ${ }^{25}$ It is thought that systemic inflammation as a consequence of systemic infection could lead to vascular endothelial cell injury, causing an intracranial haemorrhage. ${ }^{9}$ However, further investigation is needed to fully understand this concept.

Secondly, there may be a direct causal link between the actions of infectious organisms and cerebrovascular accident.1718,23,25,26 For example, bacteria that cause tuberculosis and meningitis can lead to stroke through the inflammatory injury to cerebral vessels. ${ }^{23}$ Interestingly, in the 
case of viruses, there is emerging evidence that viral load is directly linked to increased incidence of stroke. ${ }^{23}$ Initially, stroke was considered a complication of dyslipidaemia, as a side effect of highly-active anti-retroviral therapy. However, it is now thought stroke occurs as a result of virusmediated vasculopathy. Ortiz et al. ${ }^{22}$ suggested that vasculitis is the dominating mechanism for stroke, with hypercoagulability being a supportive mechanism. ${ }^{22}$ Arterial remodelling leads to either stenosed or dilated vessels, causing ischaemic or haemorrhagic strokes, respectively. ${ }^{23}$ However, it would be prudent to remember that these initial studies have small sample sizes, and include patients with HIV, which may lead to the confounding of results. Nevertheless, stroke in patients with herpes viruses, such as varicellazoster virus and herpes simplex virus have also been associated with vasculopathy, in both adults and children when compared with agematched controls. ${ }^{23}$ The vasculopathy seen in varicella-zoster virus infection is histologically similar to giant cell arteritis, indicating some inflammatory involvement. ${ }^{23}$

The associations between infection, inflammation, and stroke are complex and remain unclear. It should be noted that many of these links are formed following retrospective cohort studies, which are weakened by recall bias and missing data. Additionally, confounding factors are often not accounted for. Therefore, further investigations are warranted, to elucidate the specific pathways employed by infective organisms.

\section{CURRENT EVIDENCE OF THE CORRELATION BETWEEN STROKE AND COVID-19}

The association between COVID-19 infection and stroke is under continued investigation as increasing reports emerge with the progression of this global pandemic. Qureshi et al. ${ }^{27}$ reported that $4.9 \%$ of patients with a confirmed diagnosis of COVID-19 are expected to have a stroke. Worryingly, the mortality rate was reported to be as high as $38.0 \%$ among these patients with COVID-19, compared with $3.2 \%$ observed in all hospitalised stroke patients. ${ }^{27}$ Interestingly, stroke has also been reported as the presenting complaint for COVID-19 infections. In an Italian study of 388 patients with COVID-19, nine presented with ischaemic stroke, with six of these patients admitted to intensive care and three admitted to a general ward. ${ }^{28}$ Additionally, both Siegler et al. ${ }^{29}$ and Yaghi et al. ${ }^{30}$ reported a higher incidence of cryptogenic stroke in patients with COVID-19. Cryptogenic stroke refers to a stroke with an underlying cause distinct from atherosclerosis or embolism. This suggests that the underlying pathological process may be novel to infection. Given this association between COVID-19 and stroke, there has been much speculation surrounding the mechanisms responsible.

The proposed mechanisms are based on four different processes, including viral neurotropism, endothelial dysfunction, coagulopathy, and inflammation. Severe acute respiratory syndrome coronavirus 2 (SARS-CoV-2) is a neuroinvasive virus, as proven by its presence in the cerebrospinal fluid of an infected patient with encephalitis. ${ }^{31}$ Viral presence was also noted in cerebral capillary endothelial cells in a patient with COVID-19 following autopsy. ${ }^{32}$ This suggests that the virus may have direct effects on cerebral vessels, possibly causing endothelial dysfunction, which could lead to stroke. Endothelial dysfunction may also be mediated by angiotensin-converting enzyme 2 (ACE2) receptor binding. The binding of the virus to ACE2 receptors allows the virus to enter cells. These receptors are expressed on endothelial cells in the brain and regulate cerebral blood flow thus, viral-receptor binding may disrupt these regulatory processes, ultimately causing a stroke. ${ }^{33}$

Coagulopathy or a hypercoagulable state has also been associated with COVID-19. Elevated levels of D-dimer, fibrin degradation products, and fibrinogen were detected in patients with COVID-19, while antithrombin and prothrombin levels were reduced, indicating greater coagulation during infection. ${ }^{32}$ The concentration of procoagulant markers correlated with the severity of COVID-19, suggesting that the risk of stroke is directly proportional to infection severity. ${ }^{32}$ ACE2 receptor binding may also cause thrombosis. There is higher expression of ACE2 receptors in the heart and lungs, resulting in greater viral load at these sites. A greater immune response in the lungs can lead to hypoxia. Hypoxia is a lack of oxygen supply which generally occurs 
through endothelial injury or blood stasis; two constituents of Virchow's triad, thus initiating thrombosis. $^{34}$ Similarly, an increased immune response at the coronary vessels may lead to atherosclerotic plaques being disrupted, causing plaque rupture and subsequent thrombosis. Both these processes would lead to ischaemic stroke. Furthermore, it is well known that severe infections can be complicated by disseminated intravascular coagulation which could play a part in the onset of ischaemic stroke in patients with COVID-19.35 Another characteristic of severe COVID-19 infection is a cytokine storm, one aspect of an exaggerated immune response. This cytokine storm includes increased levels of IL-6, which has been implicated in the REasons for Geographic And Racial Differences in Stroke (REGARDS) study with increased risk of stroke, regardless of other stroke risk factors. ${ }^{36}$

These proposed mechanisms include direct effects of the virus as well as pathological systemic changes that may predispose to stroke. Given the complexity of the pathways involved in infection and inflammation, it would be expected that more than one of these proposed mechanisms is involved in the pathogenesis of stroke following COVID-19 infection. It would be prudent to investigate further in order to prevent cerebrovascular complications and continue the downwards trend in stroke mortality.

\section{MANAGEMENT OF CONCURRENT INFECTION AND STROKE}

Despite the worryingly high mortality rates reported in stroke patients with concurrent COVID-19 infection, there are no universal guidelines for the management of stroke in these patients. Present prophylaxis and treatments are predominantly based on what we understand already about stroke and infectious disease as separate entities, rather than when they occur simultaneously. ${ }^{37,38}$

Stroke may be the presenting complaint for those with COVID-19 infection; ${ }^{39}$ therefore, guidelines recommend all potential stroke patients presenting to the emergency department are screened for COVID-19.40-42 Use of telemedicine to assess patient stroke profile should be encouraged in order to minimise risk of transmission to hospital staff. ${ }^{27,42}$
Imaging investigations should be started as soon as possible, preferably within the first hour, to reduce mortality and achieve maximal functional recovery. ${ }^{27,38,42}$ CT, CT angiography, and CT perfusion scanning may be performed. ${ }^{27,41,42}$ Indeed, patients with COVID-19 commonly underwent CT and MRI across a series of case studies. $^{39,43,44}$ Baracchini et al. ${ }^{41}$ proposed the usage of a mobile CT scanner for patients with suspected or confirmed COVID-19, in accordance with infection control advice, to minimise further exposure to SARS-Cov-2. Referral for intravenous thrombolysis or mechanical thrombectomy is made on a case-by-case basis, as long as benefits outweigh the risks. ${ }^{27,42}$

Intravenous thrombolysis is effective if administered within the first 3 hours after the cerebrovascular event. Nonetheless, studies have shown that it is not appropriate for the management of ischaemic stroke in patients with infective endocarditis, raised C-reactive protein, or raised D-dimer levels, as it may increase the risk of intracranial bleeding. ${ }^{27,45}$ Interestingly, COVID-19 has been shown to elevate D-dimer levels. ${ }^{28,46}$ Thus, thrombolysis should be carefully considered in infected patients, especially since hepatic function may be impaired, reducing the clearance of thrombolytic agents. ${ }^{27}$ Poor et al. ${ }^{47}$ have reported favourable results of thrombolysis for the treatment of pulmonary thrombi in patients who are COVID-19 positive, but there is insufficient evidence that thrombolysis would be as effective in the treatment of ischaemic stroke. ${ }^{27}$

The American Stroke Association (ASA) recommends the use of mechanical thrombectomy in the case of internal carotid artery or middle cerebral artery occlusion with symptom onset of less than 6 hours and a National Institutes of Health Stroke Scale (NIHSS) score above six. ${ }^{48}$ However, two recent randomised controlled trials were able to demonstrate good efficacy of mechanical thrombectomy for up to 24 hours for patients that have had a large vessel occlusion in the anterior circulation. Patients need to fulfil eligibility criteria based on neuroimaging results, but special consideration should be given to those with a confirmed COVID-19 diagnosis given the risk of severe complications. ${ }^{48,49}$ Of those who receive thrombectomy, 38\% receive anaesthesia and of these, $14 \%$ require intubation due to complications such as respiratory failure. ${ }^{27}$ One can expect that would be the case for 
most COVID-19-positive patients experiencing respiratory symptoms. Additionally, the risk of transmission to medical staff is high.

Antiplatelet drugs, such as aspirin and clopidogrel, as well as anticoagulants (mainly enoxaparin), have been used to treat acute stroke in patients with COVID-19.39,41,43,44 No studies have reported whether antiplatelet therapy is superior to anticoagulants or vice versa, and some patients are prescribed dual therapy. ${ }^{41}$

Several papers mention the use of low-molecularweight heparin at prophylactic doses in patients with COVID-19. ${ }^{28,36,38,41-44}$ Its effectiveness, however, remains questionable; Klok et al. ${ }^{38}$ reported that despite enoxaparin administration, $31 \%$ of patients had thrombotic complications. The same study proposed that a more aggressive approach should be implemented, by increasing the frequency of administration or dose. ${ }^{38}$

\section{FUTURE RESEARCH}

The true extent of the impact COVID-19 has had on stroke will be revealed by the findings from the ongoing CASCADE study. This study aims to identify clinical predictors of stroke in patients positive for COVID-19, providing insight into the pathological pathways at play in the disease process. ${ }^{50}$

Ultimately, a greater understanding of SARSCoV-2 pathogenesis is required, in order to develop preventative measures and targeted therapies. $^{51}$ Multiple theories have been formulated in regard to the correlation between COVID-19 and stroke, but further research is needed before these theories can be translated to changes in clinical practice. The implication of inflammatory processes and production of
IL-6, as well as the role of ACE2 depletion, have been proposed as possible explanations for a causal relationship..$^{14,52}$ It is advisable to pursue the confirmation or rejection of these theories. Additionally, introducing neurological monitoring of patients may assist in the formulation of a timeline of disease progression in those with neurological sequelae. ${ }^{52}$

At present, two randomised controlled trials are reviewing the efficacy of enoxaparin as prophylactic therapy. ${ }^{53,54}$ However, in order to develop prophylaxis and treatment guidelines, more research is needed. Further trials to investigate the aforementioned proposal of increasing the dosage of low-molecular-weight heparin are needed to ensure that its benefits outweigh the risks. ${ }^{38,55}$ Lastly, a study comparing anticoagulant and antiplatelet therapies would be valuable to clinicians. Future retrospective and case studies should consider incorporating the decision-making process when choosing the best treatment for patients.

\section{CONCLUSION}

In this succinct review, stroke was discussed from an infectious disease perspective. Addressed were the potential pathophysiological mechanisms of stroke in patients with COVID-19, and how these can be used to inform decisions regarding both prophylaxis and treatment. The authors acknowledge that the scarcity and inconsistency of previous literature presents additional challenges when attempting to understand the complex relationship between stroke and COVID-19. Ultimately, further research is needed to enhance our understanding of both the direct effects and systemic repercussions of infection.

\section{References}

1. Sacco RL et al. American Heart Association Stroke Council, Council on Cardiovascular Surgery and Anesthesia. Council on Cardiovascular Radiol-ogy and Intervention. Council on Cardiovascular and Stroke Nursing et al. An updated definition of stroke for the 21st century: a statement for healthcare professionals from the American Heart Association/ American Stroke Association. Stroke.
2013;44(7):2064-89.

2. Avan A et al. Socioeconomic status and stroke incidence, prevalence, mor-tality, and worldwide burden: an ecological analysis from the Global Bur-den of Disease Study 2017. BMC Med. 2019;17:191

3. Stroke association. Stroke statistics. 2018. Available at: https://www.stroke. org.uk/sites/default/files/state_of_ the_nation_2018.pdf. Last accessed: 23rd July 2020.

4. Grau AJ et al. Common infections and the risk of stroke. Nat Rev Neurol. 2010;6(12):681-94.

5. Jillella DV, Wisco DR. Infectious causes of stroke. Curr Opin Infect Dis. 2019;32(3):285-92.

6. Ellul MA et al. Neurological 
associations of COVID-19. Lancet Neurol. 2020;DOI: 10.1016/S14744422(20)30221-0.

7. Trejo Gabriel y Galán JM. Stroke as a complication and prognostic factor of COVID-19. Neurología (English Edition). 2020;35(5):318-22.

8. Doheim MF et al. Association between Helicobacter pylori infection and stroke: a meta-analysis of 273,135 patients. J Neurol. 2020;DOI:10.1007/ s00415-020-09933-x.

9. Fugate $\mathrm{J}$ et al. Infectious causes of stroke. Lancet Infect Dis. 2014;14:86980.

10. Clayton TC et al. Recent respiratory infection and risk of cardiovascular disease: case-control study through a general practice database. Eur Heart J. 2008;29(1):96-103.

11. Lucchese $\mathrm{G}$ et al. Cross-reactivity as a mechanism linking infections to stroke. Front Neurol. 2019;10:469.

12. Kunze A et al. Recent infection as a risk factor for intracerebral and sub-arachnoid haemorrhages. Cerebrovasc Dis. 2000;10(5):352-8.

13. Epstein $\mathrm{S}$ et al. Infection and atherosclerosis: emerging mechanistic para-digms. Circulation 1999;100(4):e20-8.

14. Hess D et al. COVID-19-related stroke Transl Stroke Res. 2020;11(3):322-5.

15. Van der Poll T et al. Activation of coagulation after administration of tu-mour necrosis factor to normal subjects. N Engl J Med. 1990;322(23):1622-7.

16. Manousakis $\mathrm{G}$ et al. The interface between stroke and infectious disease: in-fectious diseases leading to stroke and infections complicating stroke. Curr Neurol Neurosci Rep. 2009:9(1):28-34.

17. Huber SA et al. Interleukin-6 exacerbates early atherosclerosis in mice. Ar-teriscler Thromb Vasc Biol. 1999;19(10):2364-7.

18. Amlie-Lefond $C$ et al. varicella zoster virus: a common cause of stroke in children and adults. J Stroke and Cerebrovasc. 2016;25(7):1561-9.

19. Rivers RPA et al. The endotoxininduced coagulant activity of human mon-ocytes. Br J Haemoatol. 1975;30(3):311-6.

20. Esmon CT et al. Inflammation and coagulation: linked processes potentially regulated through a common pathway mediated by protein C. Thromb Haemost. 1991;66(1):160-5.

21. Hesselvik JF et al. Protein C, protein $\mathrm{S}$ and $\mathrm{C} 4 \mathrm{~b}$-binding protein in severe infection and septic shock. Thromb Haemost. 1991;65(2):126-9.

22. Ortiz G et al. Mechanisms of ischaemic stroke in HIVinfected patients. Neu-rology.
2007;68(16):1257-61.

23. Miller EC et al. Infection and stroke: an update on recent progress. Curr Neurol Neurosci Rep. 2016;16(1):2.

24. Grau AJ et al. Recent infection as a risk factor for cerebrovascular ischaemia. Stroke. 1995;26(3):373-9.

25. Sebastian $\mathrm{S}$ et al. Infection as a stroke trigger. Stroke. 2019;50(8):2216-8.

26. Shindler-Itskovitch $\mathrm{T}$ et al. Helicobacter pylori infection and prevalence of stroke. 2018;24(1):e12553.

27. Qureshi A et al. Management of acute ischemic stroke in patients with COVID-19 infection: report of an international panel. Int J Stroke. 2020;15(5):540-54.

28. Lodigiani $C$ et al. Venous and arterial thromboembolic complications in COVID-19 patients admitted to an academic hospital in Milan, Italy. Throm-bosis Res. 2020;191:9-14.

29. Siegler JE et al. Cerebrovascular events and outcomes in hospitalised pa-tients with COVID-19: The SVIN COVID-19 Multinational Registry. Int J Stroke. 2020; 1747493020959216. [Epub ahead of print]

30. Yaghi S et al. SARS-CoV-2 and stroke in a New York healthcare system. Stroke. 2020;51(7):2002-11

31. Moriguchi T et al. A first case of meningitis/encephalitis associated with SARS-coronavirus-2. Int J Infect Dis. 2020;94:55-8.

32. Reddy ST et al. Cerebrovascular disease in patients with COVID-19: a re-view of the literature and case series. Case Rep Neurol. 2020;12(2):199-209

33. Paniz-Mondolfi A et al. Central nervous system involvement by severe acute respiratory syndrome coronavirus-2 (SARS-CoV-2). J Med Virol. 2020;92(7):699-702.

34. Gupta $\mathrm{N}$ et al. The stimulation of thrombosis by hypoxia. Thromb Res. 2019:181:77-83.

35. Van der Poll T et al. Coagulation and sepsis. Thromb Res. 2016;149:38-44.

36. Jenny NS et al. Inflammatory cytokines and ischaemic stroke risk: the RE-GARDS cohort. Neurology. 2019:92(20):2375-84.

37. Orsucci $D$ et al. Neurological features of COVID-19 and their treatment: a review. Drugs Context. 2020;9:20205-1.

38. Klok F et al. Incidence of thrombotic complications in critically ill ICU patients with COVID-19. Thromb Res. 2020;191:145-7.

39. Avula A et al. COVID-19 presenting as stroke. Brain Behav Immun. 2020;87:115-9.

40. Mao L et al. Neurologic manifestations of hospitalized patients With Coro-navirus Disease 2019 in Wuhan, China. JAMA Neurol. 2020;77(6):1-9.

41. Baracchini $\mathrm{C}$ et al. Acute stroke management pathway during coronavirus-19 pandemic. Neurol Sci. 2020;41(5):1003-5.

42. National Institute for Health and Care Excellence (NICE). Clinical guide for the management of stroke patients during the coronavirus pandemic. 2020. Available at: https://www. nice.org.uk/Media/Default/About/ COVID-19/Specialty-guides/Specialtyguide-Stroke-and-coronavirus.pdf Last ac-cessed: 7th January 2020.

43. Morassi $\mathrm{M}$ et al. Stroke in patients with SARS-CoV-2 infection: case series. J Neurol. 2020;267(8):2185-92.

44. Beyrouti R et al. Characteristics of ischaemic stroke associated with COVID-19. J Neurol Neurosurg Psychiatry. 2020;jnnp-2020-323586.

45. Robinson $T$ et al. Thrombolysis in acute ischaemic stroke: an update. Ther Adv Chronic Dis. 2011;2(2):119-31.

46. Grasselli et al. Pathophysiology of COVID-19-associated acute respiratory distress syndrome: a multicentre prospective observational study. Lancet Respir Med. 2020;S2213-2600(20)30370-2

47. Poor HD et al. COVID-19 critical illness pathophysiology driven by diffuse pulmonary thrombi and pulmonary endothelial dysfunction responsive to thrombolysis. Clin Transl Med 2020;10(2):e44.

48. Ren Z et al. Indications for mechanical thrombectomy - too wide or too narrow? World Neurosurg. 2019;127:492-9.

49. Mokin $M$ et al. Indications for thrombectomy in acute ischemic stroke from emergent large vessel occlusion (ELVO): report of the SNIS Standards and Guidelines Committee. J Neurointerv Surg. 2019;11(3):215-20.

50. Abootalebi et al. Call to Action: SARS-CoV-2 and CerebrovAscular Disor-dErs (CASCADE). J Stroke Cerebrovasc Dis. 2020;29(9):104938.

51. Ntaios $\mathrm{G}$ et al. Characteristics and outcomes in patients with COVID-19 and acute ischemic stroke: The Global COVID-19 Stroke Registry. Stroke. 2020:10.1161/STROKEAHA.120.031208.

52. Whittaker A et al. Neurological manifestations of COVID-19: a systematic review and current update. Acta Neurol Scand. 2020;142(1):14-22.

53. Faculdade de Medicina de Ribeirão Preto. Full versus prophylactic heparinization for the treatment of severe forms of SARS-Covid-19: clinical, randomized, open and controlled study - HeSAcovid trial. U1111-12514283. http://www.ensaiosclinicos.gov. $\mathrm{br} / \mathrm{rg} / \mathrm{RBR}-949 \mathrm{z} 6 \mathrm{v} /$.

54. CHU de Saint Etienne. Evaluation of 
the concentration-effect relationship of enoxaparin for thromboembolic prevention in COVID-19 resuscitation patients. COV-ENOX study. EudraCT
2020-001823-15. https://www. clinicaltrialsregister.eu/ctr-search/ trial/2020-001823-15/FR.

55. Abou-Ismail $M$ et al. The hypercoagulable state in COVID-19: incidence, pathophysiology, and management. Thromb Res. 2020;194:101-15. 